on MDI and their average HbA1c values when on CSCI a year later. When repeated in 2019, there was an average improvement in HbA1c levels of $48 \%$ and at 24 months an average of $53 \%$ improvement.

Conclusion This data is utilised to demonstrate to staff and patients how CSCI alone is no guarantee for normalising poorly controlled HbA1C levels. We must continue to take a holistic approach to the counselling and management of our type 1 diabetes paediatric patients.

\section{G426(P) DELAYED OR ABSENT? USE OF NEXT GENERATION SEQUENCING DIAGNOSTIC TOOLS IN A UK PUBERTY COHORT}

HR Patel, SR Howard, T Saengkaew, L Dunkel. Endocrinology, William Harvey Research Institute, London, UK

\subsection{6/archdischild-2020-rcpch.368}

Background and Aim Isolated delayed puberty (DP) and congenital hypogonadotropic hypogonadism $(\mathrm{cHH})$ are causes of severe delayed puberty that are almost clinically indistinguishable. Treatment is condition-specific and time-sensitive, yet existing methods to differentiate the two lack sensitivity and specificity. Genetic testing has been proposed as a potentially accurate diagnostic investigation. This project aimed to broaden our understanding of the genetic basis of severe delayed puberty to aid clinical diagnosis and prognosis of individual patients.

Methods Phenotype and genotype data of a UK cohort of 70 (probands $=24$, relatives $=46$ ) was studied. Whole exome sequencing was performed on probands and variants were filtered to identify candidate genes. Sanger sequencing was used to confirm variants and assess segregation with trait in pedigrees. Published literature, bioinformatics databases and prediction software tools were used to identify likely deleterious variants.

Results Following whole exome sequencing of 24 probands, 27 variants were identified in 21 candidate genes. 10 of these variants were found to be likely deleterious by prediction tools and 1 was proven to be definitively deleterious by previously published literature. 5 candidate genes had previously identified variants associated with $\mathrm{cHH}, 1$ candidate gene had variants previously associated with isolated DP and another candidate gene had variants previously associated with both phenotypes. Clinical diagnoses was established in $46 \%$ of probands. After genetic testing, a further 38\% had a likely pathogenic variant consistent with a diagnoses of $\mathrm{cHH}$ or isolated DP.

Conclusion A likely genetic diagnoses was established in 69\% of probands with previously unclassified severe delayed puberty. Thus, identification of gene mutations known to cause $\mathrm{cHH}$ or isolated DP helps to clarify diagnoses. Genetic testing has the potential to allow time-sensitive, condition-specific treatment and future planning for individual patients. Though clinical significance of variants remains uncertain, SEMA6A, SEMA3E and HS6ST1 were amongst candidate genes highlighted as potentially pathogenic providing plenty of scope for future study.

\section{G427(P) ABSTRACT WITHDRAWN}

\section{G428(P) THE 50 MOST INFLUENTIAL STUDIES ON PAEDIATRIC DIABETIC KETOACIDOSIS: A BIBLIOMETRIC ANALYSIS}

M Nandakumar, MM Maturana, E Vouzonis. Department of Paediatrics, North Middlesex University Hospital, London, UK

\subsection{6/archdischild-2020-rcpch.369}

Objectives Diabetic ketoacidosis (DKA) in paediatrics is a common condition and often the first presentation of type 1 diabetes mellitus in children. The number of citations a study receives can be used as a marker of academic influence, and can help to identify strong and weak areas of research within a particular field. We aimed to identify the 50 most influential studies relating to paediatric DKA by citation number, and analyse their characteristics.

Methods The Web of Science database was used to determine the studies that were most frequently cited. We searched for studies that included 'Diabetic ketoacidosis' or 'DKA' along with 'paediatric' or 'children' or 'child' or 'infant' in the title. Data extracted included publication year, article type and focus, publication journal, institution and country, level of evidence (LOE) and total citation number. Published guidelines were excluded from the LOE rankings. Citation density was also calculated as number of citations per year since publication.

Results Our search returned 410 studies. The top 50 studies were published between 1976 to 2014. The most common study topics were epidemiology and pathophysiology, with many studies relating to cerebral oedema $(n=19)$. The mean number of citations was 74.7 (standard deviation 60.3, range 28-375). These were published in 21 journals, led by Pediatric Diabetes $(\mathrm{n}=9)$. They originated from 9 different countries, with institutions from the United States of America publishing the majority $(\mathrm{n}=30)$, and the University of California, Davis publishing the most studies $(n=7)$. One RCT was identified, 39 non-randomised clinics studies were included. The remainder included reviews, basic science studies and guidelines. 10 studies were classified as LOE 1, and 11 studies as LOE 4.

Conclusions The 50 top cited studies on paediatric DKA relate mostly to epidemiology and pathophysiology. Cerebral oedema in paediatric DKA patients has received increased attention by researchers and institutions. LOE was low, with only one RCT identified. Though these studies are the most influential in the field, perhaps the evidence base for this field would benefit from further high quality trials.

\section{G429(P) DIAGNOSIS OF DIABETES MELLITUS IN CHILDREN, INCLUDING FACTORS LEADING TO DELAYED DIAGNOSIS}

M Thomas, A Ali. Paediatrics Department, Northwick Park Hospital, London, UK

\subsection{6/archdischild-2020-rcpch.370}

Aims To identify patient demographics, presentation and contributing factors towards delay in new diagnosis of diabetes mellitus in children, and to compare this to results from a similar audit in 2013.

Methods A retrospective audit of electronic records of all patients identified from the local paediatrics diabetes caseload database with new diagnoses between 1st January and 31st December 2018. Inclusion criteria included age at diagnosis 